\title{
Fatores de fortalecimento para a cultura de inovação em bibliotecas: proposta de checklist para autoavaliação
}

\author{
Pâmela Savedra \\ AEROTD Faculdade de Tecnologia, Florianópolis, SC, Brasil \\ pamelads20@gmail.com \\ Ana Clara Cândido \\ Universidade Federal de Santa Catarina, Departamento de Ciência da Informação, Florianópolis, SC, \\ Brasil \\ acc.anaclara@gmail.com \\ Mariene Alves do Vale \\ Universidade Federal de Santa Catarina, Departamento de Ciência da Informação, Florianópolis, SC, \\ Brasil \\ marieneavale@gmail.com
}

DOI: https://doi.org/10.26512/rici.v13.n3.27581

Recebido/Recibido/Received: 2019-10-14

Aceitado/Aceptado/Accepted: 2020-08-04

Resumo: O ambiente da biblioteca vem adaptando-se de acordo com as novas tecnologias, de maneira que as práticas possibilitem que o usuário contemple a biblioteca para além do local padronizado, que oferece o ambiente, estantes, empréstimo de livros, quietude e leitura, percebendo-a como um ambiente moderno e transformador. No entanto, sabe-se que muitas bibliotecas não utilizam o seu potencial na íntegra, sob a visão de um conceito ultrapassado. Diante disso, o presente estudo busca conhecer fatores para a autoavaliação da cultura de inovação em biblioteca. Trata-se de um estudo de natureza qualitativa, caracterizada como exploratória e descritiva. Foi realizada uma pesquisa para a elaboração de uma proposta de elementos que podem servir para fortalecer hábitos que contribuam com inovação. Como resultado, foi desenvolvido um checklist com o auxílio de profissionais da área, que serviu como instrumento de coleta de dados, permitindo que os gestores pudessem avaliar o potencial dos incentivos para o fortalecimento da cultura de inovação nas bibliotecas onde atuam.

Palavras-chave: Inovação. Biblioteca. Cultura organizacional.

Strengthening factors for the culture of innovation in libraries: proposal for a self-assessment checklist Abstract: The library environment is adapting according to the new technologies, so that the practices allow the user to contemplate the library beyond the standardized place, which offers the environment, shelves, book lending, quietude and reading, realizing it as a modern and transformative environment. However, it is known that many libraries are used improperly, under the vision of an outdated concept. Therefore, the present study seeks to know the factors for the self-evaluation of the innovation culture in the library. It is a qualitative study, characterized as exploratory and descriptive. A research was carried out to elaborate a proposal of elements that can serve to strengthen habits that contribute to innovation. As a result, a checklist was developed with the assistance of professionals in the field, which served as a data collection tool, allowing managers to assess the potential of incentives to strengthen the innovation culture in the libraries where they operate.

Keywords: Innovation. Library. Organizational culture.

Factores de fortalecimiento de la cultura de la innovación en las bibliotecas: propuesta de una lista de verificación de autoevaluación 
Resumen: El ambiente de la biblioteca se ha ido adaptando a las nuevas tecnologías, por lo que las prácticas permiten al usuario contemplar la biblioteca más allá de la ubicación estandarizada, que ofrece el entorno, estanterías, préstamos de libros, quietud y lectura, notándola como un ambiente moderno y transformador. Sin embargo, es bien sabido que muchas bibliotecas no utilizan plenamente su potencial, desde la perspectiva de un concepto desactualizado. Ante esto, el presente estudio busca conocer los factores para la autoevaluación de la cultura de innovación en las bibliotecas. Este es un estudio cualitativo, caracterizado como exploratorio y descriptivo. Se realizó una investigación para elaborar una propuesta de elementos que puedan servir para fortalecer los hábitos que contribuyen a la innovación. Como resultado, se desarrolló una lista de verificación con la ayuda de profesionales en el área, que sirvió como un instrumento de recopilación de datos, permitiendo a los gerentes evaluar el potencial de incentivos para fortalecer la cultura de innovación en las bibliotecas donde operan.

Palabras clave: Innovación. Biblioteca. Cultura organizativa.

\section{Introdução}

Nos dias correntes, a sociedade vive em constante aceleração, o que exige a existência de meios mais cômodos para obter informações, visto que ela está no alicerce da atual era da informação e do conhecimento. As tecnologias geraram hábitos novos no comportamento informacional dos usuários, de forma que a informação autêntica não está mais conectada aos recursos informacionais oferecidos pela biblioteca.

Uma unidade de informação sustenta seu propósito por meio de seu acervo e serviços de informação. Os serviços dispostos devem auxiliar no atendimento às demandas de informação da comunidade usuária da unidade.

Assim, para que atendam à demanda do usuário, as informações necessitam estar organizadas, obedecendo a parâmetros estruturados, a fim de que possam contribuir da melhor forma em todos os seus setores, que devem funcionar como um organismo interligado, respeitando os rumos da atualidade e buscando crescimento dia após dia (RANGANATHAN, 1931).

O ambiente da biblioteca vem se adaptando às novas tecnologias, de maneira que as práticas possibilitem o usuário contemplá-la para além do local padronizado, que oferece o ambiente, estantes, empréstimo de livros, quietude e leitura, de modo que ele a perceba como um ambiente moderno e transformador.

Diante da tecnologia, as bibliotecas se encontram frequentemente instigadas a inovar de forma eficaz, mantendo-se atualizadas de maneira a satisfazer as necessidades dos usuários. Assim sendo, a opção por processos de inovação é primordial, sendo decisiva na busca pelos resultados esperados.

A relevância desta pesquisa se dá pelo fato de a biblioteca ser um espaço de aprendizagem em suporte físico e digital, no qual a pesquisa, a investigação, o pensamento, a imaginação e a criatividade são fundamentais para o percurso do conhecimento e para o crescimento pessoal, social e cultural. 
No entanto, sabe-se que muitas bibliotecas são utilizadas não na sua amplitude potencial, sob a visão de um conceito ultrapassado. Portanto, familiarizar os fatores de fortalecimento para a cultura de inovação em bibliotecas se justifica pela importância de captar e manter novos usuários.

Nesse sentido, o objetivo do presente estudo é propor um checklist para autoavaliação da cultura de inovação nas bibliotecas.

\section{Cultura organizacional e inovação}

A cultura é inerente à natureza humana, em que os indivíduos participam tanto no papel de influenciadores, como sendo induzidos a criar e recriar. Schein $(2009$, p. 8) apresenta que "a cultura é intrínseca a nós como indivíduos, além de estar em constante evolução à medida que nos reunimos e criamos novos grupos que, por fim criam novas culturas".

A mudança cultural diz respeito, inicialmente, aos hábitos e comportamentos de uma comunidade, grupo ou sociedade. A exemplo do surgimento do computador, houve uma mudança significativa no modo de pensar e viver das pessoas, pois as tecnologias da informação e comunicação permitem que todos fiquem conectados de maneira global, podendo conhecer e experimentar novas culturas sem nem mesmo sair de casa.

Como consequência, os indivíduos vão se adaptando conforme os novos tempos, contribuindo para inovações e transformações. Da mesma forma que a sociedade e os grupos apresentam sua cultura, as organizações constituem suas próprias culturas organizacionais.

Para Robbins, Judge e Sobral (2010, p.501), a cultura organizacional corresponde "a um sistema de valores compartilhado pelos membros de uma organização que a diferencia das demais", tendo como características a atenção aos detalhes, a orientação para os resultados, o foco na pessoa, o foco na equipe, a agressividade, a estabilidade e, justamente, a inovação.

Assim, a cultura é formada por elementos de importância para as organizações, cujas definições estabelecem o que é cultura organizacional, como os colaboradores devem desempenhar sua rotina de tarefas, como devem se portar no ambiente interno e externo e qual a estratégia mais favorável para atingir os melhores resultados.

Assim, cada organização determina sua cultura tendo como objetivo o melhor para exercer sua função. Diante dessa percepção, a cultura organizacional é compreendida como um fator relevante para o desenvolvimento da inovação, que pode ser estimulado ou inibido pelas práticas dela decorrentes.

No que tange ao termo inovação, pode-se mencionar o Manual de Oslo como sendo um documento consensual de padronização desse conceito, definido como "um produto ou processo novo ou aprimorado (ou uma combinação destes) que difere significativamente dos 
produtos ou processos anteriores da unidade e que é disponibilizado para usuários em potencial (produto) ou utilizado pela unidade (processo)" (ORGANIZAÇÃO PARA A COOPERAÇÃO E DESENVOLVIMENTO ECONÔMICO, 2018, p. 20, tradução nossa).

A prática de inovar está vinculada ao conceito de mudança e abrange quatro principais tipos (TIDD; BESSANT; PAVITT, 2008).

A inovação de produto trata de alterações relevantes em algum produto já existente no mercado, ou criação de um novo produto que agregue valor aos usuários. A biblioteca Reanimation Library, Brooklyn, em Nova lorque, tem o acervo formado por vendas de bazar, lixões municipais, doações, livros usados e mercado de pulgas. A biblioteca tem a missão de construir uma coleção de recursos que inspiram a produção de trabalhos criativos (REANIMATION LIBRARY, 2020).

A imaginação criativa Reanimation Library conseguiu resgatar o valor de livros considerados "descartáveis" ao revivê-los difundindo sua relevância para a história e para as gerações contemporâneas, além de salientar que, mesmo com poucos recursos, é possível inovar e empreender.

A inovação de processo refere-se às alterações relevantes em processos, fluxos de desenvolvimento de um produto ou novas formas de entregar um serviço, visando, por exemplo, o aumento de produtividade ou a redução de custos. Kista, a biblioteca pública de Estocolmo, na Suécia, foi construída em um shopping, conta com design e arquitetura moderna e tecnologias digitais, como telas com informações da unidade e de seus funcionários (DANISH AGENCY FOR CULTURE, 2020). A biblioteca oferece uma gama de possibilidades, entre elas: acesso a jornais do mundo todo e a diversas revistas digitais; um café, palco e auditório para atividades culturais; salas de estudos; e computadores com acesso gratuito à internet.

A inovação de posição representa uma alteração relevante na maneira com que o produto ou serviço é inserido no mercado, podendo ser reposicionado para outra área ou setor. A Biblioteca Municipal Centenário, em Poços de Caldas, Minas Gerais, inovou ao instigar jovens a serem influenciadores de leitura, booktubers, conhecidos como criadores de conteúdo de leitura para YouTube (CASA DA ÁRVORE, 2019). Diante dessa iniciativa, a biblioteca passou a disponibilizar câmeras, refletores, microfones, editores de vídeos e cenários para os usuários usarem e criarem conteúdo, de forma a unir a leitura à modernidade, dado que a internet modificou o mundo.

A inovação de paradigma representa alterações relevantes nos modelos mentais do negócio, impactando, assim, no modo com que o produto ou serviço é consumido. A biblioteca pública na cidade de Sacramento, na Califórnia, passou a fazer empréstimo de diversos itens não convencionais em bibliotecas, como videogames, jogos de tabuleiros, máquinas de costura, 
máquinas para a confecção de bottons, mesa digitalizadora, e, em 2015, ganhou o título de "biblioteca das coisas" (FRANCO, 2018, p. 7).

Dentro do contexto em que a inovação está inserida, ainda existe a classificação referente ao grau de novidade envolvido. Nesse caso, a inovação pode ser incremental ou radical (TIDD; BESSANT; PAVITT, 2008). Sendo assim, inovação não representa separadamente um resultado do conhecimento tecnológico, mas é consequência do compartilhamento e da interação de conhecimentos explícitos e tácitos.

\subsection{Fatores para a cultura de inovação}

A cultura organizacional é essencial para a inovação. Por um lado, ela pode caracterizarse como uma barreira (MORAES et al., 2017), quando "[...] os valores, as normas e as práticas enfatizam o status quo" (GOMES; MACHADO; ALEGRE, 2015, p. 107). Em contrapartida, ela engloba elementos que podem servir para fortalecer hábitos que contribuam com a inovação, ou seja, esta deve ser assistida de um ambiente organizacional adequado, seja fabricando produtos ou prestando serviços.

Os recursos e técnicas administrativas preveem orientações para assumir padrões de liberdade individual, aceitação de erros, fomento ao risco, menos rigor nos controles processuais e nas estruturas hierárquicas e trabalhos por projetos e equipes. Isso visa um melhor inter-relacionamento de todo o grupo, e por meio desse estilo gerencial as pessoas se sentem à vontade para pôr em prática e experimentar ideias inovadoras (NASCIMENTO; COUTO; BASTOS, 2000).

A criatividade faz parte do processo de inovação, e pode ser compreendida como um processo pessoal ou grupal, que concebe ideias e pontos de vista para determinadas questões. Nas palavras de Valentim (2008, p. 4), "a criatividade é pensar algo original e a inovação é a execução, ou seja, a inovação é a implantação da idéia [sic] criativa".

Nessa mesma direção, Marcial (2016, p. 45) discorre que "A criatividade é um valor que está indissoluvelmente associada à inovação". As organizações têm de se adaptar para viabilizar liberdade suficiente para permitir a busca por possibilidades criativas, no entanto, é necessário controle para gerenciar a inovação de maneira eficaz e eficiente.

A inovação deve ser explorada de modo que a organização possa desfrutar dos benefícios, no entanto é fundamental uma gestão atenta para seus ganhos e controle de risco e incertezas (SILVEIRA; VIANNA; CÂNDIDO, 2017).

Os elementos que proporcionam a direção da inovação são peças que constituem o diagnóstico do potencial inovador. Do mesmo modo que para a gestão de empresas inovadoras, 
o diagnóstico é constituído por oito elementos: estratégia, liderança, financeiro, processo, estrutura, pessoas, cultura e relacionamentos (SILVEIRA; VIANNA; ENSSLIN, 2018).

Desta forma, torna-se essencial o vínculo da gestão e da liderança ao ambiente informacional. O papel do bibliotecário amplia-se de modo que, enquanto gestor, "é necessário desenvolver habilidades e competências gerenciais. Elas são primordiais para que o profissional consiga desempenhar a função de líder no seu dia a dia com a equipe" (SILVA, 2009, p. 76).

As práticas de gestão em bibliotecas crescem em progressão de maneira a permitir resultados positivos aos usuários da informação e ao trabalho dos próprios gestores, além de promover qualidade dos serviços internos e externos.

\subsection{Práticas de gestão e inovação em bibliotecas}

O constante crescimento tecnológico ocasiona mudanças no perfil dos usuários das unidades de informação, que atualmente "querem informação que possibilite o acesso rápido e a estruturação concisa, porém confiável e relevante" (TOMAÉL; ALCARÁ; SILVA, 2016, p. 17). Assim, existe a necessidade de atender os usuários, sempre procurando evoluir, ajustando os produtos e serviços às novas exigências.

A gestão de uma unidade de informação engloba planejamento estratégico, qualidade e marketing, visto que o planejamento estratégico objetiva uma análise da situação da instituição; determina objetivos e metas; elabora um plano de ação; executa o plano e controla os resultados. Destaca-se que o tripé missão, visão e valores, irá proporcionar um embasamento para o alcance de objetivos e para delinear as metas a serem atingidas.

O profissional bibliotecário, entre suas competências, em sua maioria técnicas, assume a conduta de gestor de unidades de informação para melhor atender às necessidades reais. Do mesmo modo, Silva $(2016$, p. 7) expõe que "as Bibliotecas ou Unidades de Informação assumem uma responsabilidade no cenário que vivenciamos, fazendo-se necessário utilizar-se de ferramentas administrativas para melhor gerenciar e fornecer os seus produtos e serviços informacionais".

O trabalho do bibliotecário gestor compreende o uso de ferramentas adequadas e visão ampla sobre a unidade de informação, para que sejam corretamente aplicadas. De modo a garantir benefícios significativos, deve-se ter como prioridade o planejamento estratégico e o desenvolvimento de funções e competências gerenciais.

No final da década de 1980, James já indicava tal cenário, ao enunciar que "consideráveis habilidades gerenciais precisam ser demonstradas pelo 'business information professionals' na seleção, aquisição e uso dessas informações [comerciais em formatos não tradicionais] de maneira econômica" (JAMES, 1989, p. 355, tradução nossa). 
Tais habilidades são mencionadas por Robbins, Judge e Sobral (2010) como a capacidade para tomar decisões, usar tecnologias, recrutar e motivar pessoas, agir de forma inteligente em situações inesperadas, dentre outras.

Os estudos de usuários concedem ao gestor as características daqueles que utilizam a unidade de informação, seu nível de satisfação, ou demais pontos relevantes, permitindo compreender suas necessidades e aplicar funções de gestão voltadas ao processo de melhoria contínua da unidade de informação.

Em decorrência do que foi apresentado, nota-se que os bibliotecários, devido às mudanças ocasionadas pela era tecnológica, devem desenvolver e contemplar em sua rotina as práticas de gestão, a fim de atender às particularidades dos usuários, além de aprimorar e expandir seus serviços.

Marcial (2016, p. 43) fortalece o ponto de vista em relação à sobrevivência, ao afirmar que "[...] a inovação surge como elemento decisivo na gestão da biblioteca e deve ser entendida como um fator de sobrevivência".

As bibliotecas se encontram frequentemente instigadas a inovar, mantendo-se atualizadas de maneira a satisfazer as necessidades dos usuários. Com isso, a opção por desenvolvimento de inovação é primordial, sendo decisiva na busca pelos resultados esperados.

Tendo em vista a aplicação deste estudo, torna-se importante enfatizar que as bibliotecas universitárias desenvolvem múltiplas funções e uma série de procedimentos, produtos e serviços, tornando-se organizações complexas.

Nas últimas décadas, o avanço tecnológico fez com que o seu papel tivesse que ser reavaliado, de maneira que elas pudessem oferecer serviços diferenciados.

Esse processo de renovação leva a biblioteca universitária a qualificar-se para contribuir decisivamente nas tarefas de ensino, pesquisa e extensão; tornando-se referência na busca pela informação e ideias, ou contendo-se (como ainda acontece em muitos casos) com a tarefa menor de ser apenas um depósito de livros ou uma sala de leitura e estudos opcionais.

Nessa perspectiva, a inovação manifesta-se como fator resolutivo na gestão da biblioteca, e deve ser considerada como elemento de permanência para aprimorar os produtos e serviços oferecidos por esta unidade de informação.

\section{Procedimentos metodológicos}

Esta pesquisa caracteriza-se como de natureza aplicada, pois expõe resultados acerca da situação da biblioteca universitária de uma instituição de ensino superior (IES) privada localizada em Florianópolis, em relação ao fortalecimento da cultura de inovação. 
Quanto à forma de abordagem do problema, a pesquisa é definida como qualitativa. Entende-se como pesquisa qualitativa aquela que visa explorar e entender os fatores de fortalecimento da cultura de inovação.

Com relação aos objetivos, a pesquisa é caracterizada como exploratória e descritiva, visto que pretende expandir a familiaridade e delinear fatores de fortalecimento para a cultura de inovação em bibliotecas (LAKATOS; MARCONI, 2010).

A presente pesquisa, na aplicação dos procedimentos técnicos, pode ser classificada como bibliográfica, já que faz uso de literaturas publicadas anteriormente. Além disso, pode ser considerada como um levantamento, uma vez que se faz necessária a solicitação de informações aos profissionais da área (GONÇALVES, 2005).

A biblioteca foi fundada em 2010, quando a IES despertou interesse em desenvolver e aplicar Know-how adquirido com ensino profissionalizante, em educação superior, propondo-se a implantar um curso superior de nível tecnológico.

O acervo é formado por aproximadamente 4.570 títulos e 5.182 exemplares. 0 acervo físico é organizado por estantes e o virtual encontra-se arquivado no banco de dados do sistema utilizado pela biblioteca. Ela é gerenciada a partir de seu regulamento interno e pelas normas definidas pela diretoria da IES, e desenvolve os seguintes serviços: consulta local; empréstimo domiciliar; pesquisa bibliográfica; orientação metodológica à comunidade acadêmica sobre normalização documental; acesso à rede Wi-fi; acesso ao computador; espaço para estudo; orientação metodológica; visita orientada; entre outros.

\subsection{Construção da proposta de checklist}

Como instrumento de coleta de dados foi utilizado um checklist. Para o desenvolvimento do instrumento foram utilizados como base os resultados das pesquisas bibliográficas em relação à identificação dos elementos de gestão da inovação e a contribuição de bibliotecários especialistas, por meio da participação no pré-teste do checklist.

Quadro 1 - Suporte conceitual para a construção e proposta de checklist

\begin{tabular}{|c|l|}
\hline \multicolumn{1}{|c|}{ Elemento } & \multicolumn{1}{c|}{ Autor } \\
\hline Identificação dos fatores para a cultura de & Scherer e Carlomagno (2009); Silveira \\
\hline
\end{tabular}




\begin{tabular}{|l|l|}
\hline inovação & $(2016)$ \\
\hline Ambiente de trabalho & $\begin{array}{l}\text { Marcial (2016); Tidd, Bessant e Keith } \\
\text { (2008); Valentim (2008) }\end{array}$ \\
\hline Recursos financeiros & $\begin{array}{l}\text { Marcial (2016); Tidd, Bessant e Keith } \\
(2008) ; \text { Trias De Bes e Kotler (2011); } \\
\text { Valentim (2008) }\end{array}$ \\
\hline Recursos humanos & $\begin{array}{l}\text { Bruno-Faria e Fonseca (2014); Drucker } \\
(1986) ; \text { Valentim (2008) }\end{array}$ \\
\hline Qualidade profissional & $\begin{array}{l}\text { Trias De Bes e Kotler (2011); Valentim } \\
(2008)\end{array}$ \\
\hline Serviços & Marcial (2016); Valentim (2008) \\
\hline
\end{tabular}

Fonte: Elaborado pelas autoras (2019).

Após a elaboração do checklist, a fase de pré-teste foi fundamental para a adequação do instrumento à realidade da unidade de informação. Esta etapa contou com a contribuição de três profissionais da área, bibliotecários de uma biblioteca universitária de uma IES pública, para a validação e possíveis ajustes.

Na pesquisa, foram levantados 42 elementos que auxiliam no fortalecimento da gestão de inovação em bibliotecas, divididos entre oito dimensões do Octógono da Inovação (SCHERER; CARLOMAGNO, 2009), ferramenta utilizada para o diagnóstico e gestão de empresas inovadoras. As oito dimensões são: Estratégia; Estrutura; Liderança; Cultura; Pessoas; Financeiro; Processo; e Relacionamento.

Após a validação, o checklist de autoavaliação foi respondido por um profissional responsável pela biblioteca universitária estudada. Para cada fator, foram considerados os pesos de 1 (um) a 5 (cinco), conforme classificação:

1. Insuficiente: elemento não existente na biblioteca e/ou imperceptível;

2. Fraco: elemento existe na biblioteca, porém há muito a melhorar;

3. Regular: elemento existe, atende à necessidade; mas está longe do ideal;

4. Bom: elemento existe, conforme o esperado;

5. Excelente: elemento existe, e está acima do esperado.

\section{Resultados da aplicação em biblioteca universitária}

Entre as oito dimensões avaliadas no instrumento de pesquisa, quatro foram consideradas como pontos fortes, a saber, pessoas, estratégia, cultura e liderança. Uma foi considerada como ponto fraco, a estrutura. As três restantes, processo, relacionamento e 
financeiro, encontram-se em uma faixa regular. As pontuações obtidas na avaliação podem ser observadas no Gráfico 1.

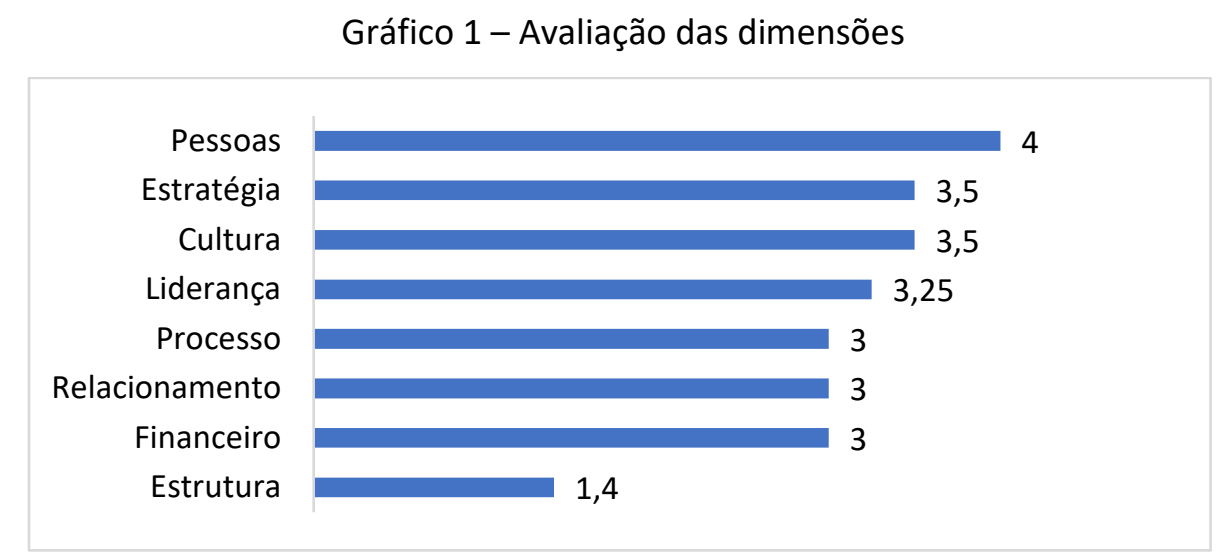

Fonte: Elaborado pelas autoras (2019).

A seguir são apresentados os resultados das dimensões que se destacaram, como ponto fraco e como pontos fortes.

Como ponto fraco, a biblioteca tem uma estrutura única, não estando subdividida em setores, e todas as funções são coordenadas e desenvolvidas por um profissional bibliotecário no período vespertino/noturno, de segunda a sexta-feira. Estas evidências podem ser analisadas no Gráfico 2.

\section{Gráfico 2 - Dimensão Estrutura}

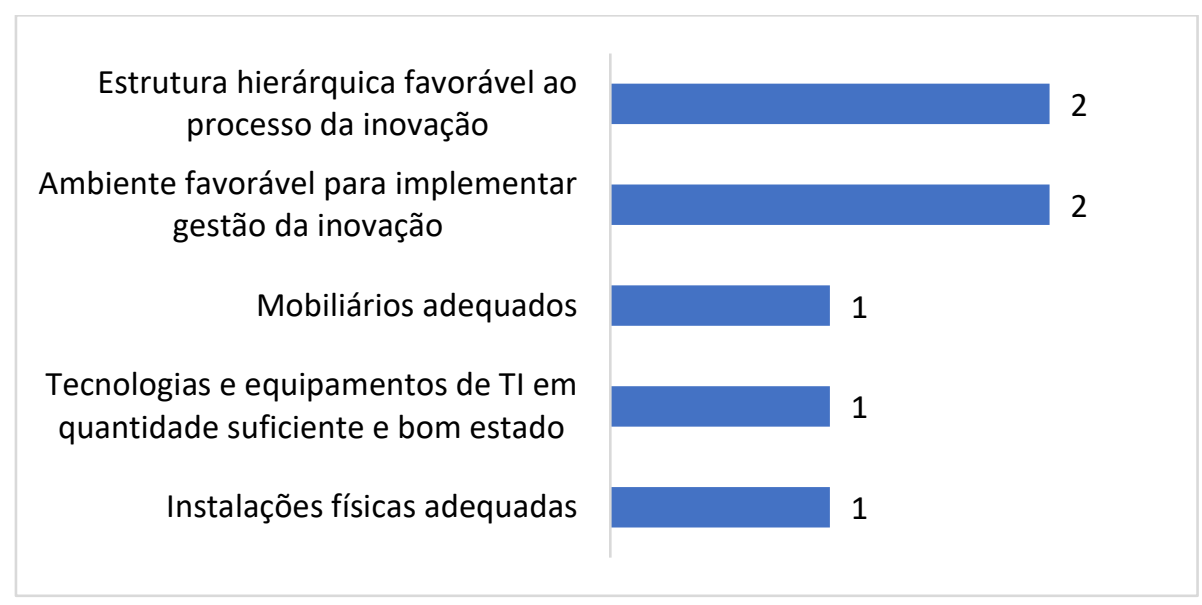

Fonte: Elaborado pelas autoras (2019).

O mobiliário não é adequado, e a biblioteca não conta com recursos de tecnologia da informação (TI) em quantidade e em bom estado (para a operacionalização da biblioteca). A 
insuficiência de infraestrutura e equipamentos modernos, no sentido de inovar em serviços, torna a estrutura uma barreira para a inovação da instituição.

Nesse sentido, nota-se que bibliotecas vinculadas a instituições, em grande parte, precisam do apoio da instituição para conduzir os problemas em relação ao mobiliário e TI. Sendo assim, não estimam esta atribuição diretamente à biblioteca, por não ser diretamente o órgão que decide ou soluciona estes itens (SILVEIRA, 2016).

Para que uma empresa seja inovadora, é necessário que tenha uma estrutura que possibilite a criatividade, mas não uma única estrutura que valha para todas (SCHERER; CARLOMAGNO, 2009).

\section{Gráfico 3 - Dimensão Pessoas}

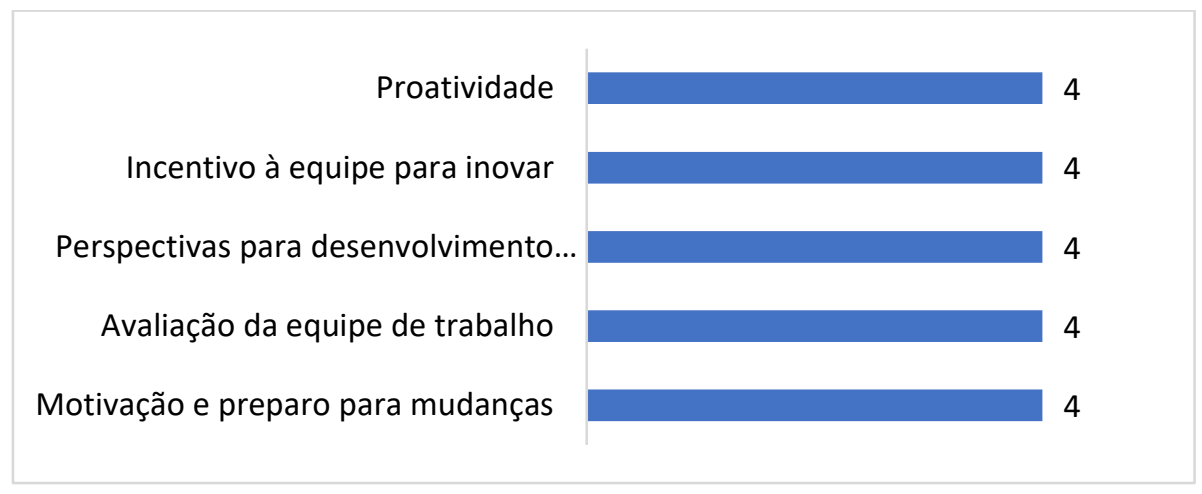

Fonte: Elaborado pelas autoras (2019).

A equipe é formada por uma bibliotecária e duas estagiárias, que são incentivadas e capacitadas para inovar e organizar o ambiente. Para além de uma boa estrutura, os colaboradores também precisam ser incentivados e estimulados a inovar, porém não existe ganho em incentivar e estimular a inovação ou possibilitar os recursos necessários para que isso seja efetivamente feito. Conforme se observa no Gráfico 3.

Para que a inovação possa existir em uma organização, cabe à equipe e a cada colaborador desempenhar o seu papel. Pensar da mesma forma faz com que as crenças tendam a ser sempre as mesmas, e a criatividade vem de ideias, conhecimentos, diversidade de perspectivas e experiências, que atuam como combustível para esse desenvolvimento da inovação (SCHERER; CARLOMAGNO, 2009). 
Gráfico 4 - Dimensão Estratégia

Análise estratégica (missão, projetos, visão de futuro)

Identificação dos pontos fracos e fortes da biblioteca

Conhecimento da cultura, práticas e éticas da biblioteca

Utilização de marketing para atrair usuários

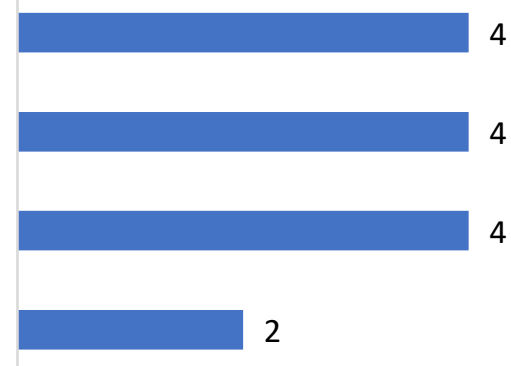

Fonte: Elaborado pelas autoras (2019).

Como estratégia, dimensão representada no Gráfico 4, a unidade tem missão, visão, valores e princípios bem definidos. Como missão, a biblioteca apresenta: disseminar a informação e torná-la acessível a toda comunidade acadêmica, criando condições para o estudo independente e a autoaprendizagem.

Sua visão é ser referência em qualidade de informação em relação ao acervo e aos serviços prestados ao usuário na área de conhecimento em que é especializada. Os valores são: ética, respeito e integridade; compromisso com serviços de qualidade; responsabilidade e eficiência.

Os princípios são descritos da seguinte forma: satisfação do usuário; qualidade dos serviços; agilidade e eficiência na prestação de informações, competência, segurança e inovação apoiados na tecnologia; formação de capacitação em aperfeiçoamento dos usuários.

Assim sendo, é atribuição dos gestores compreender e buscar estratégias para definir quais os fatores ou dimensões melhoram o funcionamento de sua organização, de modo a conduzir esses fatores ao fomento do êxito organizacional, contudo o percurso da gestão da inovação deve conter visão, estratégica, planejamento, organização e controle (SILVEIRA, 2016). 
Gráfico 5 - Dimensão Cultura

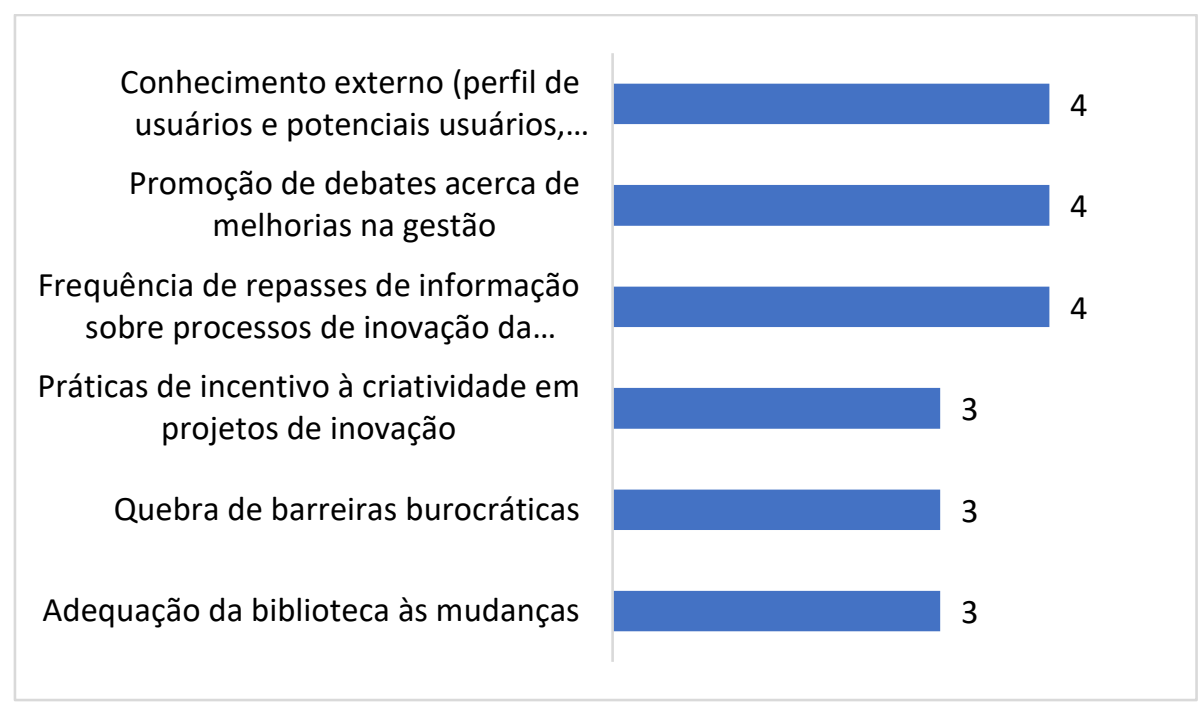

Fonte: Elaborado pelas autoras (2019).

Sobre a cultura, cujos elementos de avaliação estão dispostos no Gráfico 5, a posição da biblioteca na estrutura organizacional é clara, e sua estrutura interna e seu regulamento são bem definidos. A bibliotecária participa das reuniões semanais dos gestores da IES; acompanha as diretrizes de trabalho que vão ao encontro do projeto de desenvolvimento da instituição (PDI); conta com o apoio dos demais setores.

Compreende-se que a cultura de uma organização é algo intangível. Trata-se de normas e regras estabelecidas e aceitas que moldam o comportamento dos colaboradores, assim a dimensão cultura pertence à conduta da autogestão de criar e estimular o ambiente a inovar (SCHERER; CARLOMAGNO, 2009).

Desse modo, cada organização determina sua cultura tendo como objetivo o melhor para exercer sua função. Diante dessa percepção, a cultura organizacional é compreendida como um fator relevante para o desenvolvimento da inovação, visto que dispõe de elementos que podem servir ou inibir práticas que contribuam com a inovação.

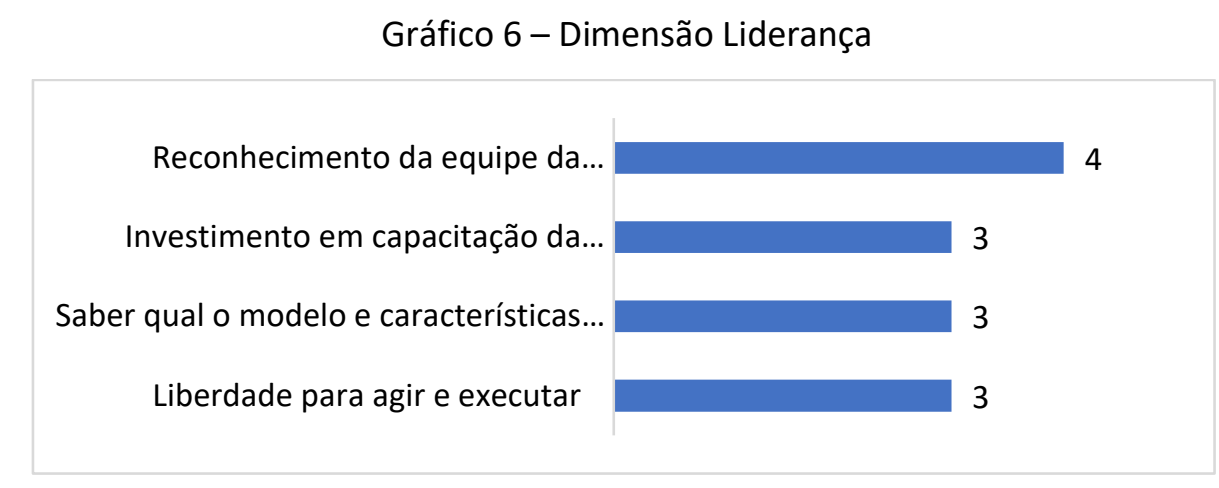

Fonte: Elaborado pelas autoras (2019). 
A liderança, dimensão apresentada no Gráfico 6, procura sempre agir e realizar ações com o princípio de assegurar a satisfação dos usuários, não só por meio de serviços que ofereçam qualidade, agilidade, confiabilidade, inovação e eficiência, apoiados na competência e tecnologia, mas também com foco em viabilizar a aquisição de materiais em bom estado de conservação, de fácil recuperação, e também promovendo a criatividade e a capacitação dos colaboradores, por meio de treinamentos e cursos de aperfeiçoamento.

A liderança, de modo geral refere-se à administração, sendo ela responsável pela estratégia da organização, por estabelecer recursos e determinar as normas competitivas e de evolução da organização.

Neste ponto, constata-se que a liderança não é somente dos altos executivos, mas, sim, de todos aqueles que, dentro de uma organização, têm habilidade de influenciar outras pessoas. Identificam-se, portanto, os considerados líderes formais e os informais (SCHERER; CARLOMAGNO, 2009).

As dimensões processo, relacionamento e financeiro foram consideradas regulares, não havendo nenhum ponto de atenção ou algo significativo para ser enaltecido.

\section{Considerações finais}

As bibliotecas enquanto instituições que auxiliam o desenvolvimento social de seu público usuário oferecem suporte informacional para suprir necessidades individuais ou coletivas de âmbito pessoal ou do meio, seja acadêmico, escolar ou outro (AMARAL, 1996). Ainda que o rumo tecnológico esteja dificultando este papel, os profissionais bibliotecários estão gerindo suas unidades de informação para que seus serviços não se tornem inválidos para com a sociedade.

O desenvolvimento do presente estudo possibilitou uma análise da importância da gestão da inovação em bibliotecas, tornando possível elencar os elementos que contribuem para práticas inovadoras, sendo possível conhecer os fatores que auxiliam na autoavaliação da cultura de inovação em bibliotecas.

A relevância da análise das dimensões e seus elementos se dá pela importância de assegurar que a inovação seja contínua, estruturada e proposital, reconhecendo as pessoas por suas ideias e enaltecendo talentos individuais, e, consequentemente, demonstrando resiliência frente às dificuldades. Desse modo, ao incentivar seus integrantes a atuar com autonomia, as instituições correspondem às demandas externas, desenvolvem novas ideias e melhoram o atendimento, ampliando sua eficiência.

Com base nos fatores identificados referentes às dimensões que auxiliam no fortalecimento da gestão de inovação em biblioteca, como resultado da pesquisa foi 
desenvolvido um checklist e aplicado na biblioteca (objeto de estudo). Entre as oito dimensões avaliadas no instrumento de pesquisa, quatro foram consideradas como pontos fortes: pessoas, estratégia, cultura e liderança; e uma como ponto fraco: estrutura; e três encontram-se em uma faixa regular: processo, relacionamento e financeiro.

Durante a análise e estudo da biblioteca, pode-se perceber pelas autoras alguns pontos relevantes. Na interpretação de síntese dos resultados procedente à análise e descrição foram conhecidas as particularidades da dimensão pessoas com a avaliação do trabalho em equipe, os incentivos para inovação, motivação e preparo para mudança, proatividade e perspectivas para desenvolvimento pessoal.

Essa foi a única dimensão com pontuação máxima em todos os itens de avaliação. Por se tratar de um instrumento de autoavaliação, aplicado à bibliotecária responsável, a atribuição de notas nessa dimensão pode ter sido enviesada, por se tratar diretamente do seu papel como gestora da unidade em relação às interações estabelecidas com a própria equipe.

Dentre as observações retiradas desta análise, a dimensão estrutura é considerada ponto fraco, por não atender aos requisitos determinados na mesma. Com base nas repostas obtidas pela bibliotecária responsável identifica-se que nesse estudo o fator primordial que precisa de melhorias é o elemento estrutura. É explícito que as finanças fornecidas à biblioteca são fortemente limitadas sem haver um orçamento próprio anual para aquisições. Intrínseco a tal questão é possível pensar que a comunicação entre o setor financeiro e biblioteca estão em discordância. Aconselha-se, portanto, a apresentação de dados fixos ao setor financeiro para que este seja sensibilizado a respeito da importância de aquisições e modificações na estrutura, investimento em mobiliários mais modernos, mais espaço para atender as normas estabelecidas para funcionamento adequado da biblioteca, um sistema menos complexo e mais dinâmico, são considerados pontos de melhoria.

Por fim, o estudo propõe apresentar uma ferramenta de autoavaliação, sendo respondida pela responsável da unidade de informação. Esta pode ser uma fragilidade, pois os resultados remetem à opinião de uma profissional, neste caso, que possui conhecimento sobre as questões estratégicas do contexto. Além do possível viés, presente nas respostas a itens que dizem respeito ao seu próprio trabalho, como os das dimensões pessoas e liderança.

No entanto, a aplicação poderia ser aprofundada e complementada com a opinião de outros colaboradores da própria unidade de informação. Além disso, um checklist adaptado poderia ser aplicado a outros interagentes, como docentes e estudantes da IES, de modo que uma avaliação paralela servisse como subsídio para a análise crítica da autoavaliação. Neste caso, seria possível abordar tópicos que não perpassam, exclusivamente, pelo funcionamento interno, mas que também são explorados na autoavaliação, como a identificação de pontos 
fortes e fracos da biblioteca, na dimensão estratégia, ou a existência de instalações físicas adequadas, na dimensão estrutura.

Dada à pertinência do assunto, sugere-se a aplicação do checklist de autoavaliação em outras instituições; avaliação dos resultados da aplicação e elaboração de planos de ação para melhorias nos pontos fracos identificados; e criação de checklists específicos para cada dimensão.

\section{Referências}

BRUNO-FARIA, Maria de Fátima; FONSECA, Marcus Vinicius de Araujo. Cultura de Inovação: conceitos e modelos teóricos. Revista de Administração Contemporânea, Rio de Janeiro, v. 18, n. 4, p. 372-396, jul./ago. 2014. Disponível em: http://www.scielo.br/pdf/rac/v18n4/1415-6555rac-18-04-00372.pdf. Acesso em: 3 abr. 2019.

CASA DA ÁRVORE. Biblioteca forma booktubers e atrai jovens leitores. Poços de Caldas, 2019. Disponível em: http://casadaarvore.art.br/biblioteca-forma-booktubers-e-atrai-jovens-leitores. Acesso em: 7 fev. 2020.

DANISH AGENCY FOR CULTURE. Model Programme for Public Libraries. Copenhague, 2020. Disponível em: https://modelprogrammer.slks.dk/en/news-events/public-library-of-the-yearprevious-years/public-library-of-the-year-2015/\#c123450. Acesso em: 7 fev. 2020.

DRUCKER, Peter Ferdinand. Inovação e espírito empreendedor (entrepreneurship): prática e princípios. São Paulo: Pioneira, 1986.

FRANCO, Isabela de Melo. Empreendedorismo e inovação: um novo perfil de bibliotecas. Múltiplos Olhares em Ciência da Informação, Belo Horizonte, v. 8, n. 2, nov. 2018. Disponível em: http://portaldeperiodicos.eci.ufmg.br/index.php/moci/article/view/3810/2189. Acesso em: 12 jun. 2019.

GOMES, Giancarlo; MACHADO, Denise D.P. N.; ALEGRE, Joaquin. Determinantes da cultura de inovação: estudo na indústria têxtil de Santa Catarina. Brazilian Business Review, Vitória, v. 12, n. 4 , p. 105-129, jul./ago. $2015 . \quad$ Disponível em: https://bbronline.com.br/index.php/bbr/article/view/135. Acesso em: 10 fev. 2020.

GONÇALVES, Hortência de Abreu. Manual de metodologia da pesquisa científica. São Paulo: Avercamp, 2005.

JAMES, Sylvia. Understanding managerial principles and practices: management training for business information professionals. Education for Information, v. 7, n. 4, p. 355-363, 1989. Disponível em: https://content.iospress.com/articles/education-for-information/efi7-406. Acesso em: 3 abr. 2019.

LAKATOS, Eva Maria; MARCONI, Marina de Andrade. Fundamentos de metodologia científica. 7. ed. São Paulo: Atlas, 2010.

MARCIAL, V. F. Inovação em bibliotecas. In: RIBEIRO, A. C. M. L.; FERREIRA, P. C. G. (org.). Biblioteca do século XXI: desafios e perspectivas. Brasília: Ipea, 2016. p. 43-59. 
MORAES, Marcela Barbosa de et al. Cultura de inovação em micro e pequenas empresas dos setores aeronáutico e de tecnologia da informação. Exacta, São Paulo, v. 15, n. 3, jul./set.., p. 441-456, 2017. Disponível em: http://www.redalyc.org/articulo.oa?id=81052980006. Acesso em: 10 fev. 2020.

NASCIMENTO, Cecilia Maria Pereira do; COUTO, Ana Maria de H. C. de Sá; BASTOS, Márcia Maria Silvestre. A biblioteca universitária hoje: gerência compartilhada. Rio de Janeiro: Universidade Federal $\quad$ Fluminense, 2000.2 Disponível em: https://app.uff.br/riuff/bitstream/1/478/1/Nascimento\%2C\%20CeciliaA\%20Biblioteca\%20universit\%C3\%A1ria\%20hoje-Evento-2000.pdf. Acesso em: 3 abr. 2019.

ORGANIZAÇÃO PARA A COOPERAÇÃO E DESENVOLVIMENTO ECONÔMICO. Oslo Manual 2018: Guidelines for Collecting, Reporting and Using Data on Innovation. Paris: OCDE, 2018. Disponível em: https://doi.org/10.1787/9789264304604-en. Acesso em 7 fev. 2020.

RANGANATHAN, S. R. The five laws of library science. Madras: Madras Library Association; London: Edward Goldston, 1931.

REANIMATION LIBRARY. Mission. Nova York, 2020. Disponível em: https://www.reanimationlibrary.org/pages/mission. Acesso em: 7 fev. 2020.

ROBBINS, Stephen P.; JUDGE, Timothy A.; SOBRAL, Filipe. Comportamento organizacional: teoria e prática no contexto brasileiro. 14. ed. São Paulo: Pearson Prentice Hall, 2010.

SCHEIN, Edgar H. Cultura organizacional e liderança. São Paulo: Atlas, 2009.

SCHERER, Felipe O.; CARLOMAGNO, Maximiliano S. Gestão da inovação na prática: como aplicar conceitos e ferramentas para alavancar a inovação. São Paulo: Atlas, 2009.

SILVA, Cícera A. M. G. A aplicabilidade do marketing de relacionamento na gestão de bibliotecas universitárias: um estudo em uma biblioteca de instituição privada de Juazeiro do Norte - CE. Folha de Rosto, v. 2, n. 2, jul./dez. 2016. Disponível em: https://periodicos.ufca.edu.br/ojs/index.php/folhaderosto/article/view/98/118. Acesso em: 3 abr. 2019.

SILVA, Janaina da Costa. 0 estilo de liderança dos bibliotecários de Brasília. 2009. Dissertação (Mestrado em Ciência da Informação) - Universidade de Brasília, Brasília, 2009. Disponível em: http://repositorio.unb.br/bitstream/10482/4944/1/2009 JanainaCostaSilva.pdf. 3 abr. 2019.

SILVEIRA, Murilo Mauro. Elementos formais para implantação da gestão da inovação em bibliotecas. 2016. Dissertação (Mestrado em Ciência da Informação) - Universidade Federal de Santa Catarina, Florianópolis, 2016.2 Disponível em: https://repositorio.ufsc.br/xmlui/handle/123456789/174289. Acesso em: 9 jun. 2019.

SILVEIRA, Murilo Mauro; VIANNA, William Barbosa; CÂNDIDO, Ana Clara. Fundamentos conceituais para abordagens de gestão da inovação em bibliotecas. Biblios: Journal of Librarianship and Information Science, University of Pittsburgh, n. 68, p. 69-81, 2017. Disponível em:

http://www.scielo.org.pe/pdf/biblios/n68/a05n68.pdfhttp://dx.doi.org/10.5195/biblios.2017. 359. Acesso em: 3 abr. 2019. 
SILVEIRA, Murilo Mauro; VIANNA, William B.; ENSSLIN, Sandra Rolim. Gestão da inovação em bibliotecas: elementos fundamentais de revisão de literatura internacional. Investigación Bibliotecológica: archivonomía, bibliotecología e información, v. 32, n. 76, p. 29-44, jul./sept. 2018. Disponível em: http://dx.doi.org/10.22201/iibi.24488321xe.2018.76.57973. Acesso em: 9 jun. 2019.

TIDD, Joe; BESSANT, John; PAVITT, Keith. Gestão da inovação. 3. ed. São Paulo: Bookman, 2008.

TOMAÉL, Maria Inês; ALCARÁ, Adriana Rosecler; SILVA, Terezinha Elizabeth da. Fontes de informação digital: critérios de qualidade. In.: TOMAÉL, Maria Inês; ALCARÁ, Adriana Rosecler. (Org..). Fontes de informação digital. Londrina: Eduel, 2016, p. 13-44.

TRIAS DE BES, Fernando; KOTLER, Philip. A bíblia da inovação: o modelo A-F. São Paulo: Leya, 2011.

VALENTIM, Marta L. P. Criatividade e inovação na atuação profissional. CRB-8 Digital, São Paulo, v. 1 , n. 1, p. 3-9, jul. 2008. Disponível em: http://www.humanativa.com.br/wpcontent/uploads/2016/08/Artigo-criatividade-e-inova\%C3\%A7\%C3\%A30.pdf. Acesso em: 3 abr. 2019. 